\title{
Microanalysis in the Pharmaceutical and Biomedical Industry
}

\author{
M.A. Miller and T.B. Vander Wood
}

MVA, Inc. 5500 Oakbrook Parkway, Suite 200, Norcross, GA 30093

Pharmaceutical and biopharmaceutical companies are frequently faced with particulate problems observed during routine Quality Assurance inspection or products returned as customer complaints. Microanalysis is useful in characterizing the contaminants to determine the origin and whether they are related to a raw material, the manufacturing process, or another type of contaminant, including adulterants. This paper highlights selected case studies of how microanalysis is used to identify particulate materials in the pharmaceutical industry and solve process related problems in the biomedical industry.

Spectral data on particles as small as $15 \mu \mathrm{m}$ makes infrared microspectroscopy (micro-FTIR) a technique that is very useful in identifying foreign materials and their sources. White particles observed in an injectable drug were isolated by filtration and analyzed by micro-FTIR. The particles were identified as a flouropolymer and were traced to a Roulon ${ }^{\circledR}$ processing component. Other foreign materials identified include polymer fragments, fibers, and common airborne contaminants, such as lint, particleboard and skin cells. In many cases, the particles have been identified as materials related to the product formulation or containers.

In another case, a manufacturer received a small piece of a tablet from a customer. Concerns over the identity of the tablet, which appeared partially dissolved with no distinguishing markings, prompted an investigation. Analysis of the extracted active ingredient showed the partial tablet was the product identified on the pharmacy label, much to the relief of the consumer, the pharmacy and the manufacturer (FIGURE 1.).

Quality Assurance inspection revealed dark spots on the surface of rubber stoppers used in a parenteral product. Microscopical examination of the dark spots revealed the spots were actually insects on the rubber (FIGURE 2.). Further analysis by electron microscopy revealed the insects were at the surface of the rubber, partly and slightly embedded at the surface. The intact morphology, together with the slightly embedded insect bodies, suggested the insects were on the surface of the mold during stopper manufacturing, and the contamination did not occur at the pharmaceutical plant.

Field emission scanning electron microscopy (FE-SEM) is a highly valuable technique in the examination of nanometer-sized structures in medical devices. A medical device manufacturer detected large variations in the deposition of radioactive iodine onto copper wire substrates. Examination of the wires at low magnification by light microscopy indicated there were no gross signs of contamination. FE-SEM revealed distinctly different surface features with regard to surface porosity. The wires with decreased porosity of the surface which corresponded to a lower surface area, also showed lower uptake of the iodine. The wires that took up a greater amount of the iodine 
had a much greater porosity. These differences in surface porosity were directly related to the electroplating and etching conditions of the copper plating.

Another medical device manufacturer was interested in imaging their implanted medical devices after removal of the devices from patients for investigation of the bacteria on the surfaces. Field emission scanning electron microscopy was used to image the regions with significant bacterial growth.

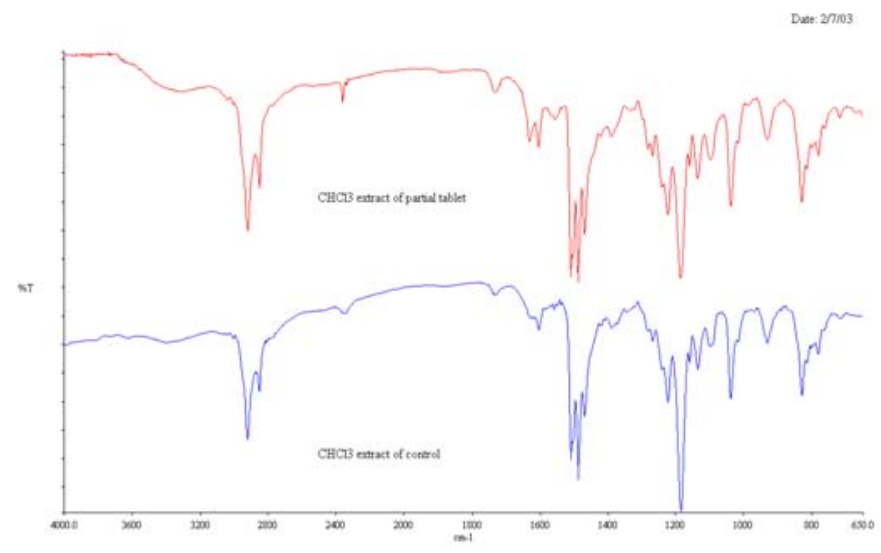

FIGURE 1. Infrared spectra of active ingredient from partial tablet and suspected product.

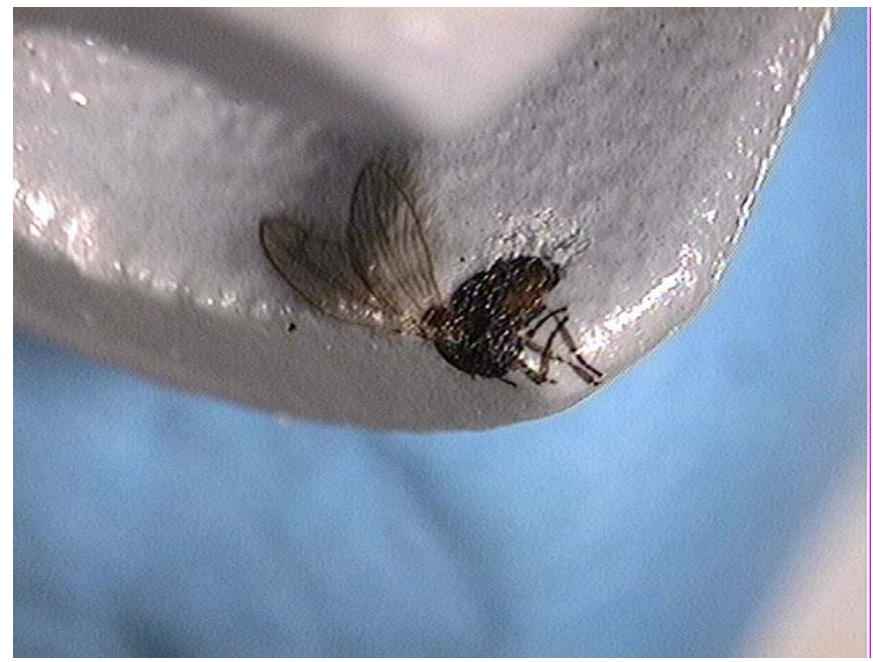

FIGURE 2. Light macrograph of "dark spot” on rubber vial stopper. 